\title{
PRÓLOGO
}

\section{SOBRE LA POSIBILIDAD DE OTROS PAISAJES: CONVENCIONES, PAISAJES, HUMO, MEMORIAS Y MONUMENTOS}

\author{
Dante Angelo ${ }^{1}$ y Eduardo Herrera-Malatesta ${ }^{2}$
}

El estudio de paisajes en la arqueología cobró un inusitado interés hace algunas décadas cuando fue planteado como una alternativa a una arqueología que -influenciada ampliamente por la geografía espacialproponía el estudio de los contextos arqueológicos desde el análisis espacial y los principios de la economía formalista. En ese entonces, contribuciones de académicos como Walter Christaller (1966) y otros se incorporan en la arqueología como una herramienta metodológica que permitía un acercamiento a aquellos espacios territoriales que habían sido ocupados en el pasado (Clarke 1977; Johnson 1977). En Latinoamérica, y en particular, en el área andina y regiones aledañas, esta preocupación puede ser remontada a esfuerzos pioneros como los de Gordon R. Willey (1953), que serían posteriormente complementados por trabajos arqueológicos de análisis espacial y regional, estudiados tanto a partir de contextos arqueológicos (Moore 1996; Netherly 1984) como a partir de evidencia material y documentos históricos (Zuidema 1962; cf. Silverman e Isbell 2002).

Los trabajos que se presentan en este espacio, gentilmente concedido por Chungara, fueron inicialmente presentados y discutidos en el marco de la XIX Reunión de Teoría Arqueológica de América del Sur (TAAS), realizado en la ciudad de La Paz, Bolivia, en Mayo del 2016. Allí, dichas contribuciones, además de algunas otras que no pudieron ser incorporadas como parte de esta publicación, respondieron al llamado de trabajar el concepto de paisajes. La convocatoria original, titulada: "Paisajes en conflicto: Procesos, sociedades, personas" buscó reflexionar sobre el paisaje como el estudio de la "complejidad de la vida de las personas, las contingencias históricas, los conflictos, el movimiento y el cambio" (Bender 2000), mediante una aproximación a los paisajes alternativos que se gestan en distintos procesos históricos.

Durante la década final del siglo pasado, el interés por el estudio de espacios y territorios se ve asaltado por la crítica post-procesual que, buscando insertar una perspectiva más humanista a estudios de arqueología regional de asentamientos y la arqueología ecológica, introduce formalmente el estudio de paisajes en arqueología (Anschuetz et al. 2001; Edmonds 1999). Así, incorporando elementos de la teoría social, el concepto de paisaje pasa a referirse a aquél espacio conformado por lugares socialmente conocidos por poseer distintas dimensiones (ritual, social, económica u otra) que articulan la vida social y política de las personas (Ashmore 2002). Si bien diversos autores trabajando en América Latina remarcan a la arqueología de paisajes como el marco conceptual apropiado para dar cuenta de aspectos referidos a ritualidad e ideología (p.ej. Moore 1996; Silverman e Isbell 2002), estos en su generalidad enfatizan aquellos aspectos funcionales del paisaje; no es sino hasta hace muy recientemente que, desde la arqueología, se ha empezado a explorar aspectos ontológicos y relacionales como es el caso de Pazzarelli y Lema (este número), en los que se entiende la relación entre seres humanos y paisajes como mutuamente constitutivos (cf. Nielsen et al. 2017).

Complementariamente, asumiendo el desafío de pensar desde Latinoamérica, se buscó cuestionar y tensionar aquellas connotaciones y perspectivas tradicionales del concepto, que generalmente privilegian el punto de vista occidental, para poder dar paso y voz a paisajes previamente relegados o marginales. En ese sentido, quizás uno de los aspectos más significativos que emergen de esta crítica está relacionado a que estos paisajes son social y contextualmente significativos pero que, "sin importar cuan diferentemente pueden ser interpretados los elementos de estos paisajes previos, siempre tienen la capacidad de influir en la mentalidad actual" (Crumley 1999:272). Como se muestra en algunos de los trabajos (Pazzarelli y Lema, y Marconetto y Bussi), nuevas miradas desde la arqueología no solamente pueden contribuir a "comprender la relación entre seres humanos y medio ambiente en términos de sus dinámicas de largo-alcance" (Erickson 2000:350),

\footnotetext{
${ }^{1}$ Departamento de Antropología, Universidad de Tarapacá, Arica, Chile. dangeloz@gmail.com

${ }^{2}$ Faculteit Archaeologie, Universitiet Leiden. Leiden, the Netherlands. e.n.herrera.malatesta@arch.leidenuniv.nl
} 
sino también incorporar en dichas relaciones la complejidad de entretejer experiencias y vivencias en paisajes cargados de historicidad.

En ese sentido, en los cuatro textos que componen este dossier, el conflicto es evidenciado y presentado en la tangibilidad e intangibilidad del paisaje. Así, por ejemplo, Marconetto y Bussi plantean una propuesta que, enmarcada en una perspectiva que incorpora lo etnográfico, plantea una crítica en torno a la percepción distópica de cambio climático como una forma de "fin del mundo". Desde esta mirada, apoyados en un andamiaje teórico recientemente propuesto por autores como Viveiros de Castro (2004; cf. Danowski y Viveiros de Castro 2014 y otros), evidencia cómo los paisajes no solamente están construidos en el espacio sino en el tiempo y la percepción de sus cambiantes estados de ánimo (clima) y las tensiones que éstos generan; así, el entender la configuración del paisaje es quizás la forma más adecuada de abordar un tema de otra manera inconmensurable como lo es entender y enfrentar el colapso. Los "fines del mundo" al que Marconetto y Bussi nos acercan no necesariamente están constituidos por esa especie de telón de fondo que determina o condiciona el accionar humano, sino -según se entiende- a visualizar mecanismos que incorporan hitos de inestabilidad y destrucción cataclística como parte de una experiencia traducida en el paisaje de Ambato.

Por otra parte, el artículo de Pazzarelli y Lema, forma parte de una nueva colaboración entre dos autores que, desde distintas vertientes de la antropología (etnografía) y arqueología (arqueobotánica) han venido trabajando temas teóricos (Pazzarelli 2017; Lema y Pazzarelli 2015) con perspectivas originales e innovadoras. Así, el artículo que se incluye en este grupo de trabajos es parte de una continua colaboración en el abordaje de perspectivas y discusiones ontológicas; aquí, los autores conjugan el análisis de experiencias que remarcan la importancia del entrelazamiento de cuerpos, materialidades y sustancias en la configuración de los paisajes en los Andes. Para Pazzarelli y Lema, el conflicto está dado en la producción misma del paisaje, ya que ésta debe ser entendida como un proceso de involucramiento en el que las equivocaciones son el habitar mismo de este paisaje.

Su propuesta aporta significativos elementos para conceptualizar paisajes arqueológicos cuando, por ejemplo, remarcan la importancia de considerar el paisaje huacheño como el resultado de un proceso relacional expresado en actividades cotidianas como lo es el mismo procuramiento de recursos (p.ej. buscar leña). Así, los paisajes no simplemente hablan de escenarios estáticos sino de las mismas actividades de la gente que los habita y les dá sentido; este es el caso de la práctica de aprovisionamiento de leña o la selección arbitraria de espacios de ritualidad relacionado a sitios arqueológicos mencionados por Pazzarelli y Lema.

Una situación similar es la que produce la presencia de las herrumbradas torres de hierro que actualmente coronan espacios de frontera menos activos que cuando fueron originalmente eregidas, en el caso de la contribución de Dante Angelo. Tomando el caso de la construcción de la frontera y sus paisajes en períodos históricos, Angelo ofrece quizás la versión más ortodoxa de estudios arqueológicos de paisajes. Su análisis se enfoca en restos materiales cuya presencia da lugar a nuevas narrativas de poder al tiempo que margina las previamente presentes. Los paisajes de frontera, en su argumento, tienen la capacidad de promover lazos identitarios que se articulan en un trasfondo político y económico que está implícito y construido en un paisaje de otra forma anodino, pero poderoso al momento de definir estructuras de pertenencia e identidad nacional.

El trabajo de Ariel Morrone explora el conflicto desde la mirada disciplinar de la historia en el caso de disputas de poder y territorio en la extensa región circundante del Lago Titicaca. Como tal, los paisajes de Morrone son casi imperceptibles ( $¡$ al punto que el autor omite la palabra paisaje en su texto!), y solo emergen en referencias a una materialidad reflejada en lugares demarcados por la acción híbrida de mojones, lagunas y otros rasgos del paisaje. Los paisajes, por tanto, están inmersos en la tensión generada por la búsqueda de control político de estos territorios y sus contestaciones o disputas y en las prácticas de uso (como la siembra de ciertos espacios). De esta forma, Morrone provee elementos útiles en la reconstrucción de paisajes coloniales e históricos que, desde la arqueología, puedan acercar cierto carácter emic al análisis de situaciones de conflicto y dinámicas de territorialidad.

En un contexto en que la tecnología parece dictar los lineamientos de las investigaciones de paisaje, a partir de sofisticados instrumentos de registro y análisis de datos, y ante una creciente adopción de herramientas metodológicas que facilitan la modelación y análisis espacial (SIG, teledetección, realidad virtual y otros) (cf. Ashmore 2000:1172), la contribución de Marconetto y Bussi y el texto de Pazzarelli y Lema sobresalen por plantear un análisis distinto de paisajes arqueológicos. En estos trabajos, los argumentos se articulan a partir de entrelazar miradas arqueológicas, en el sentido de estar basados en una observación del mundo material, con aquellas de tipo etnográfico, que informan sobre cómo este paisaje es percibido y -tomando uno de los términos empleados- "equivocado" por quienes lo habitan. 
Morrone, por otra parte, elabora su análisis a partir del estudio de documentos y archivos, que se constituyen el elemento central del análisis de un paisaje que está inmerso en el territorio que busca reconstruir históricamente.

En síntesis, el simposio organizado durante el TAAS, contó con una masiva participación y estuvo caracterizado por una apertura transdisciplinar (o no disciplinar) de la discusión; lamentamos que, por motivos de tiempo u otros compromisos previos, no todos los participantes estén representados en este volumen y agradecemos a los autores participantes. El tratamiento de paisajes, según argumenta Angelo (este número), está aún enmarcado en una mirada tradicional guiada por perspectivas funcionalistas que impiden incorporar nuevos elementos a la conceptualización de los mismos. Estamos convencidos que la tenacidad de los autores en el proceso de insistir con otros paisajes posibles de ser entendidos por la arqueología (y la antropología), es la contribución de este modesto grupo de trabajos. Esperamos, por tanto, que éstos no solo contribuyan introduciendo elementos provocadores en la discusión teórica de aquello que denominamos arqueología de paisajes, sino también a establecer esta especie de puentes interdisciplinares en el debate.

Agradecimientos: Dante Angelo agradece al comité editorial de Chungara por su apoyo y en particular Vivien Standen, Editora en Jefe, y a Eugenia Rosello, editora de producción, por su seguimiento cercano y meticuloso del proceso de edición.

\section{Referencias Citadas}

Anschuetz, K.F., R.H. Wilshusen y Ch.L. Scheick 2001. An archaeology of landscapes: Perspectives and directions. Journal of Archaeological Research 9 (2):157-211.

Ashmore, W. 2002. Decisions and dispositions': Socializing spatial archaeology. American Anthropologist 104 (4):11721183.

Bender, B. 2002. Time and Landscape. Current Anthropology 43 (S4):103-112.

Christaller, W. y C. Whiteford Baskin 1966. Central Places in Southern Germany. Prentice-Hall, Englewood Cliffs.

Clarke, D.L. 1977. Spatial Archaeology. Academic Press, London.

Crumley, C.L. 1999. Sacred Landscapes: Constructed and Conceptualized. En Archaeologies of Landscape: Contemporary Perspectives, editado por W. Ashmore y A. Bernard Knapp, pp. 269-276. Blackwell, Oxford.

Danowski, D. y E. Viveiros de Castro 2014. Há Mundo por Vir? Ensaio sobre os Medos e os Fins. Ed. Instituto Socioambiental, Río de Janeiro.

Edmonds, M. 1999. Ancestral Geographies of the Neolithic: Landscapes, Monuments and Memory. Routledge, London.

Erickson, C. 2000. The Lake Titicaca Basin: A Precolumbian built landscape. En Imperfect Balance: Landscape Transformations in the PreColumbian Americas, editado por D. Lentz, pp. 311356. Columbia University Press, New York.

Johnson, G.A. 1977. Aspects of Regional Analysis in Archaeology. Annual Review of Anthropology 6 (1):479-508.
Lema, V.S. y F. Pazzarelli 2015. Memoria Fértil. Crianza de la Historia en Huachichocana. Nuevo Mundo. Mundos Nuevos. http://journals.openedition.org/nuevomundo/67976; DOI: 10.4000/ nuevomundo.67976

Moore, J.D. 1996. The archaeology of plazas and the proxemics of ritual: Three Andean traditions. American Anthropologist 98 (4):789-802.

Netherly, P.J. 1984. The management of Late Andean irrigation systems on the North Coast of Peru. American Antiquity 49 (2):227-254.

Nielsen, A., C. Angiorama y F. Ávila 2017. Ritual as interaction with non-humans: Pre-Hispanic mountain pass shrines in the Southern Andes. En Rituals of the Past. Prehispanic and Colonial Case Studies in Andean Archaeology, editado por S. Rosenfeld y S. Bautista, pp. 241-266. University Press of Colorado, Boulder.

Pazzarelli, F. 2017. A Sorte Da Carne. Topologia Animal Nos Andes Meridionais. Horizontes Antropológicos 48:129-149

Silverman, H. y W. Isbell (eds.) 2002. Andean Archaeology II: Art, Landscape, and Society. Springer, New York.

Viveiros de Castro, E. 2004. Perspectival Anthropology and the Method of Controlled Equivocation. Tipití: Journal of the Society for the Anthropology of Lowland South America (1):3-22.

Willey, G.R. 1953. Prehistoric Settlement Patterns in the Viru; Valley, Peru. Smithsonian Institution. Bureau of American Ethnology. Washington, D.C.

Zuidema, R.T. 1962. The Ceque System of Cuzco: The Social Organization of the Capital of the Inca. Brill Archive, Leiden. 